\title{
Precision study of radio emission from air showers at LOFAR
}

Olaf Scholten ${ }^{1,2, a}$, Antonio Bonardi ${ }^{5}$, Stijn Buitink ${ }^{3}$, Arthur Corstanje ${ }^{5}$, Ute Ebert ${ }^{6,7}$, Heino Falcke $^{4,5}$, Jörg Hörandel ${ }^{5}$, Pragati Mitra ${ }^{3}$, Katharine Mulrey ${ }^{3}$, Anna Nelles ${ }^{8}$, Jörg Rachen ${ }^{5}$, Laura Rossetto ${ }^{5}$, Casper Rutjes ${ }^{6}$, Pim Schellart $^{9}$, Satyendra Thoudam ${ }^{10}$, Gia Trinh ${ }^{1}$, Sander ter Veen ${ }^{1}$, and Tobias Winchen ${ }^{3}$

1 University of Groningen, KVI Center for Advanced Radiation Technology, 9747 AA Groningen, The Nether-
lands
${ }^{2}$ Vrije Universiteit Brussel, Dienst ELEM, IIHE, Pleinlaan 2, 1050 Brussels, Belgium
${ }^{3}$ Astrophysical Institute, Vrije Universiteit Brussel, Pleinlaan 2, 1050 Brussels, Belgium
${ }^{4}$ Netherlands Institute for Radio Astronomy (ASTRON), 7990 AA Dwingeloo, The Netherlands
${ }^{5}$ Department of Astrophysics/IMAPP, Radboud University, P.O. Box 9010, 6500 GL Nijmegen, The Nether-
lands
${ }^{6} \mathrm{CWI,} \mathrm{Centrum} \mathrm{Wiskunde} \mathrm{\&} \mathrm{Informatica,} \mathrm{Amsterdam,} \mathrm{The} \mathrm{Netherlands}$
${ }^{7}$ TU/e, Eindhoven University of Technology, Eindhoven, The Netherlands
${ }^{8}$ Physics and Astronomy, University of California, Irvine, CA 92697-4575,U.S.A
${ }^{9}$ Department of Astrophysical Sciences, Princeton University, Princeton, NJ 08544, USA
${ }^{10}$ Department of Physics and Electrical Engineering, Linnéuniversitetet, 35195 Växjö, Sweden

\begin{abstract}
Radio detection as well as modeling of cosmic rays has made enormous progress in the past years. We show this by using the subtle circular polarization of the radio pulse from air showers measured in fair weather conditions and the intensity of radio emission from an air shower under thunderstorm conditions.
\end{abstract}

The paper emphasizes the measurement of circular polarization of the radio pulse from air showers as a great technical achievement, without explaining what its source is. It is stated that the main polarization is linear from the transverse current in the shower front (presumably from the opposite bending of electrons and positrons in the geomagnetic field) and the polarization of the Askaryan radiation is radially polarized. Is circular polarization somehow a coherent combination of these two? Whatever the case, its source and significance should be described.

\section{Introduction}

We will show that even the finest details of radio emission, such as its circular polarization, are well understood. Thus the detecting the radio emission from extensive air showers (EASs) as induced by energetic cosmic rays is understood to the extent that it can be regarded as a very sensitive way to determine shower properties, such as energy $[1,2]$ and $X_{\max }$, the atmospheric (slant) depth where the number of air-shower particles reaches a maximum $[3,4]$. These shower properties are used in turn to infer the nature of the primary cosmic ray, in particular its mass $[5,6]$. We are now even at the stage

\footnotetext{
ae-mail: Scholten@KVI.nl
} 

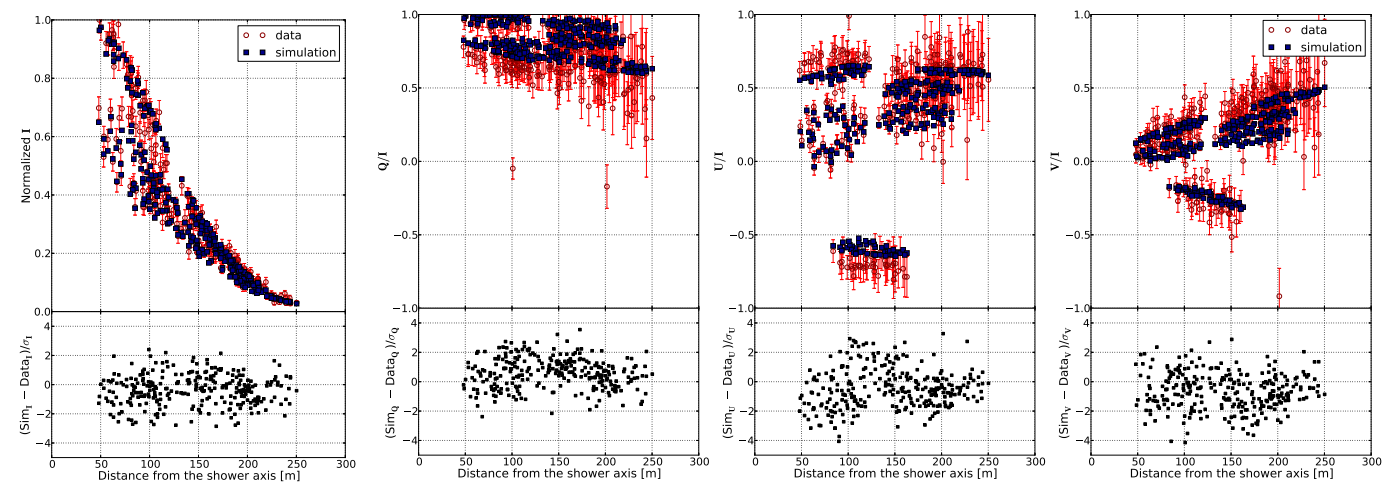

Figure 1. The intensity footprint and normalized Stokes parameters of an air shower as recorded with the LOFAR low-band antennas and projected into the shower plane (open red circles) is compared to the results of a CoREAS simulation (filled blue squares). $\sigma$ denotes one standard deviation error.

where radio emission can be used to learn about shower development in the atmosphere and thus -for example- strength and direction of atmospheric electric fields [7].

To do so, detailed models have been developed that calculate the radio emission from the EAS based on the motion of individual electrons and positrons in the air shower. Two such microscopic models are CoREAS [8] and ZHAireS [9]. Both successfully reproduce the features of the radio emission [4]. The signal has a dominant linearly-polarized component along the direction of the Lorentz force, $\hat{e}_{\vec{v} \times \vec{B}} \propto \hat{e}_{\vec{v}} \times \hat{e}_{\vec{B}}$, due to the induced transverse current in the shower front [10]. Here the shower direction is given by $\hat{e}_{\vec{v}}$ while $\hat{e}_{\vec{B}}$ denotes the direction of the geomagnetic field. A secondary contribution, also known as Askaryan radiation [11], is due to the build-up of excess negative charge in the shower front [12]. This Askaryan radiation is radially polarized and thus leads to the prediction that the deviation from the main polarization direction depends on the viewing angle, in good agreement with observations [13-17].

\section{Circular polarization in fair-weather events}

A very complete analysis of the polarization data of radio pulse as measured at LOFAR for fair weather events has been made in Ref. [16, 18], using the Stokes parameters $I, Q, U$, and $V$. In terms of the Stokes parameters the linear polarization angle with the $\vec{v} \times \vec{B}$-axis is obtained as $\psi=\frac{1}{2} \tan ^{-1}(U / Q)$, while $V / I$ specifies the circular polarization.

In Fig. 1 the measured values of the Stokes parameters for one particular event are show as function of distance to the shower axis. The data are compared with the results of a CoREAS simulation showing an excellent agreement between the two, not only for the intensity $I$, but in particular also for $V / I$, the amount of circular polarization. The circular polarization of the radio pulse can be understood by regarded it as the superposition of two separate pulses, one due to geomagnetic and one to charge excess, that have a relative time-delay and a different polarization direction. As a result the polarization vector rotates over the duration of the pulse giving rise to a finite value for $V / I$.

Since the dominant polarization of the pulse is in the $\vec{v} \times \vec{B}$-direction the ratio of $U / I$ and $V / I$ as given in Fig. 1 can be used to extract the time-delay as explained in detail in Ref. [18]. One observes that at a distance of about $100 \mathrm{~m} \mathrm{V/U} \approx 0.3$ for antennas at an angle close to $90^{\circ}$ with respect to the $\vec{v} \times \vec{B}$ axis (corresponding to extreme values for $U$ and $V$ ). Since at $100 \mathrm{~m}$ from the 
axis the signal is dominated by coherent Cherenkov emission the pulse is very narrow in time, thus wide in frequency. In the measured frequency band $30-80 \mathrm{MHz}$, with mean frequency of $50 \mathrm{MHz}$, a value $V / U=0.3$ corresponds to an arrival time difference of approximately $\Delta t=1 \mathrm{~ns}$ for the two polarization directions [18].

To understand the difference in the timing of the radio pulse emitted through the two mechanisms requires more subtle arguments [18]. For the transverse current contribution the emitted radiation relates to the vector potential as $\vec{E}_{t c}=-d \vec{A} / d t$ while for the charge excess contribution $\vec{E}_{c e}=-d A^{0} / d r$. Since the different components of the vector potential have a very similar dependence on retarded time $\left(t_{\text {ret }}=Z / c\right.$ where $z$ is the distance along the shower axis to the impact point on Earth) and $d t_{\text {ret }} / d t \approx c(R / r) d t_{\text {ret }} / d r$ [10], where $R$ is the distance to the emission point, we can see that for transverse current emission the lower parts of the shower (small $R$ ) are weighted less as compared to charge excess emission. This results in a mean emission height difference of about $1 \mathrm{~km}$ translating to an arrival time difference at $d=100 \mathrm{~m}$ of $1 \mathrm{~ns}$, in agreement with the data.

\section{Thunderstorm events}

An interesting application of using the measured radio-footprint to infer the structure of the currents in the shower is given in Ref. [7] where this technique is used to determine the structure of the atmospheric electric fields. The polarization direction determines the direction of the fields while the circular polarization tells about the change of orientation of the fields.
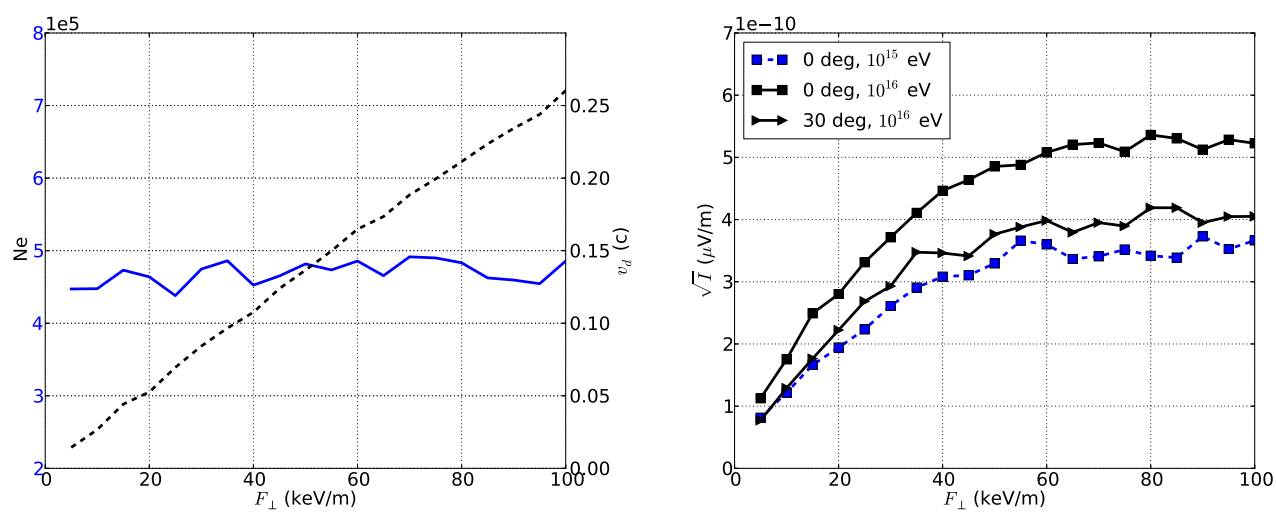

Figure 2. The left hand shows the number of electrons (solid blue line, left axis) and their drift velocity at $X_{\max }$ (dashed black line, right axis) of vertical $10^{15} \mathrm{eV}$ showers as a function of the net-transverse forces. On the right the square root of the power $\sqrt{I}$ in the frequency interval of $30-80 \mathrm{MHz}$ at the ring of maximal intensity is shown for vertical $10^{15} \mathrm{eV}$ showers (dashed line) as well as for vertical and inclined $10^{16} \mathrm{eV}$ showers (solid lines) as a function of the net-transverse force. For the $10^{16} \mathrm{eV}$ showers the $\sqrt{I}$ is scaled down by a factor 10 .

The amplitude of the radiation (square-root of the intensity) is expected to be proportional to the induced current in the shower front which is the product of the number of charged particles and the drift velocity. As is shown in the left hand plot in Fig. 2, with increasing transverse field the number of charged particles (solid blue line, left axis) remains almost constant while the drift velocity (dashed black line, right axis) shows an almost linear increase. This dependence continues up to a rather large field strength of $100 \mathrm{kV} / \mathrm{m}$. The intensity of the radiation as shown on the right of Fig. 2 
shows that only for small fields the thus expected linear increase of the amplitude is observed in the CoREAS simulations while for electric fields exceeding $(E=50 \mathrm{kV} / \mathrm{m})$ the amplitude predicted by the simulation saturates [19].

This counter-intuitive dependency can be understood by investigating the structure of the showers in more detail as is done in Ref [19]. Particularly important in this respect is the structure of the shower front, the pancake. With increasing transverse drift velocity the longitudinal component of the velocity has to decrease as the total velocity of the particles should remain below c, the light velocity. With decreasing longitudinal velocity the particles trail further behind the shower front thus increasing the pancake thickness. Since the radiation of the pancake can only be coherent for wavelength exceeding its thickness [20] the radiation lacks coherence for the frequency interval under consideration. The increase of the current with increasing field strength is thus compensated by the loss of coherence resulting in an amplitude that even drops for very large fields. An immediate consequence of this is that at lower frequencies the amplitude remains sensitive to the field [19].

\section{Conclusions}

In recent years much progress has been made in the understanding of Radio emission from air showers. Examples of this are subtle circular polarization of air showers in fair weather conditions and the less subtle saturation of the amplitude for showers exposed to rather large thunderstorm electric fields. Because of this high level of understanding, radio emission can be used as a diagnostic tool for the determination of air showers parameters such as $X_{\max }[3,6]$ and atmospheric electric fields [7].

\section{References}

[1] A. Nelles et al., J. Cosm. and Astrop. Phys. 05, 018 (2015); arXiv:1411.7868.

[2] A. Aab et al., Phys. Rev. D 93, 122005 (2016); arXiv:1508.04267.

[3] S. Buitink et al., Phys. Rev. D 90, 082003 (2014).

[4] T. Huege, Phys. Rep. 620, 1 (2016); arXiv:1601.07426.

[5] W.D. Apel et al., Phys. Rev. D 90, 062001 (2014).

[6] S. Buitink et al., Nature 531, 70 (2016).

[7] P. Schellart et al., Phys. Rev. Lett 114, 165001 (2015)

[8] T. Huege, M. Ludwig, and C. James, AIP Conf. Proc. 1535, 128 (2012).

[9] J. Alvarez-Muñiz et al., Astropart. Phys. 35, 325 (2012).

[10] O. Scholten, K. Werner, and F. Rusydi, Astropart. Phys. 29, 94 (2008); K. Werner and O. Scholten, Astropart. Phys. 29, 393-411 (2008).

[11] G.A. Askaryan, Sov. Phys. JETP 14, 441 (1962).

[12] K.D. de Vries et al., Astropart. Phys. 34, 267 (2010).

[13] V. Marin, for the CODALEMA Collaboration, Proc. 32 ${ }^{\text {nd }}$ ICRC, Beijing, 1, 291 (2011).

[14] H. Schoorlemmer, for the Pierre Auger Collaboration, Nucl. Instr. and Meth. A 662, S134 (2012).

[15] A. Aab et al., Phys. Rev. D 89, 052002 (2014); arXiv:1402.3677.

[16] P. Schellart et al., J. Cosm. and Astrop. Phys. 1410, 014 (2014); arXiv:1406.1355.

[17] A. Bellétoile et al., Astropart. Phys. 69, 50 (2015)

[18] O. Scholten et al., Phys. Rev. D 94, 103010 (2016); arXiv:1611.00758.

[19] T.N.G. Trinh et al., Phys. Rev. D 93, 023003 (2016), arXiv:1511.03045.

[20] Olaf Scholten, Krijn D. de Vries, Klaus Werner, Nucl. Instr. and Meth. A 662, S80 (2012). 